\title{
Application of MIH for the lightweight deployment of LTE-advanced systems through mobile relaying
}

\author{
Jorge Cabrejas*, Pablo Gualda, Jose F Monserrat and David Martín-Sacristán
}

\begin{abstract}
In a conventional cellular network end users connect directly to a Base Station (BS). Mobile relaying allows establishing an indirect two-hop link between the end user, called Mobile Node (MN), and the BS through a Mobile Relay (MR). This spreads out the cell coverage and increases the cell-edge throughput hence improving fairness among nodes. This article is focused on a Long Term Evolution Advanced (LTE-A) cellular network where MNs and MRs are connected through a Wireless Fidelity (WiFi) ad-hoc connection. It is proposed the use of Media Independent Handover $(\mathrm{MIH})$ signaling to define an efficient dynamic routing mechanism for MR in this framework. The proposed mechanism, called MIH-Driven Relay Selection Mechanism (MIDRES), detects which is the best direct or indirect link with the BS based on information collected using MIH messages. The MNs or MRs send MIH messages when experiencing bad channel conditions, that is detected thanks to predefined thresholds. Then, the BS starts a polling process, again supported by MIH signaling, and performs optimal route selection either through the LTE-A radio interface or through a WiFi ad-hoc interface. This article examines the implementation of this mechanism and obtains the optimal thresholds that maximize operational performance. Moreover, the potential benefit of this LTE-compliant mobile relaying solution is evaluated using a calibrated simulation tool. The results show significant savings in cost of network deployment.
\end{abstract}

Keywords: mobile relaying, MIH, LTE, LTE-advanced

\section{Introduction}

The volume of data traffic has increased significantly in recent years. In fact, in December 2009 for the first time in history, the volume of worldwide voice traffic was below data traffic [1]. Extrapolating this trend, it is estimated that by 2020 data traffic will be the dominant in mobile communications primarily due to the proliferation of applications like video streaming. As a solution to this demand, new technologies such as Long Term Evolution Advanced (LTE-A) [2] or Worldwide Interoperability for Microwave Access (WiMAX) IEEE 802.16m have emerged as promising standards. These technologies are designed to deliver peak data rates above $1 \mathrm{Gbps}$ with bandwidths of up to $100 \mathrm{MHz}$ for low mobility users and 100Mbps for high speed users [3]. However, high data rates can only be provided within the vicinity of Base Station (BS).

\footnotetext{
* Correspondence: jorcabpe@iteam.upv.es

Universitat Politècnica de València, Camino de Vera S/N, Valencia 46022, Spain
}

Therefore, it would take a large number of BSs to cover a given area with high data rates. Obviously, this implies a deployment cost that operators cannot afford. This situation motivated the study and development of relays as a mean to increase the coverage indoor scenarios and public transportation vehicles (trains, buses, etc).

The use of fixed cooperative relays has already been introduced in WiMAX mobile [4]-Institute of Electrical and Electronic Engineers (IEEE) 802.16m standard-and in the Third Generation Partnership Project (3GPP) LTE-A standard. Although the results are valid for both systems, this article is focused on the 3GPP solution.

A further step towards full integration of relaying is the use of mobile terminals as relays, which is known as mobile relaying. Xiao et al. [5] studied the usage of Mobile Relays (MRs) to extend coverage and increase throughput. Results showed an increase in coverage ranging from $21 \%$ to $44 \%$ depending on the distance from the Mobile Node $(\mathrm{MN})$ to the BS, and an increase in maximum throughput

any medium, provided the original work is properly cited. 
from $20 \%$ to $60 \%$ depending on the transmission power and the path loss exponent. In [6], Vanganuru et al. proposed the use of a hybrid wireless network with direct radio links between MNs. The BS chooses the best relay to maximize the throughput of each radio link. This mechanism results in average throughput gains of $40 \%$ assuming capacity is calculated with Shannon formula and regardless of the cost of signaling in the selection of the best relay.

Concerning the motivation of users to collaborate as MRs, there is a lot of literature addressing different ways to reinforce this cooperation (see e.g., [7]). Among other strategies, [7] presents the idea of reputation, in which users cooperate to increase their status and, consequently, be aided in the moments in which they need the support of another MR.

However, the optimal relay selection is of great importance and signaling overhead cannot be underestimated. An optimal routing mechanism must be defined so as it can dynamically adapt to changes in the system while involving the least possible signaling overhead. To date, the LTE-A standard only contemplates the use of fixed relays and lack any mechanism of MR selection. Within this framework, this article proposes the use of IEEE 802.21 Media Independent Handover (MIH) standard to support the relay selection process. MIH standard is an application protocol that can be easily implemented on mobile and network devices. MIH defines a set of entities and messages that facilitate the interchange of information between network nodes. It allows to have local and remote reports on link status so that decisions can be made dynamically in the access network. Besides, it provides intelligence to both the physical and medium access control (MAC) layers and network information to upper layers [8]. Initially, this standard was designed to optimize handovers in heterogeneous networks. In [9], Bae et al. proposed to use MIH signaling to support a triggering mechanism for the management of vertical handovers based on the data rate. On the other hand, Seol and Chung proposed to conduct vertical handover between LTE and WiMAX by adding some new nodes in the core network with a MIH function entity (MIHF) [10]. The work from Bultmann et al. [11] addressed handover signaling and introduced discovering capacity and some procedures for handover in heterogeneous networks assuming LTE assisted with fixed relays. It is worth noting that $\mathrm{MIH}$ signaling was only used to make handovers between BSs of different networks rather than to select relays.

This article proposes a new application of MIH signaling to the management of multiple radio interfaces in a cellular system assisted with MRs. The system scenario comprises an LTE-A network where MNs have another WiFi radio interface for ad-hoc communications. This assumption is fully aligned with current technological trends since both radio interfaces are expected to coexist in coming mobile devices. This article aims at analyzing the signaling requirements and provides not only an optimal configuration but also mechanisms for reducing the overhead. Moreover, we analyze to what extent mobile relaying can support the deployment of lightweight networks.

The rest of the article is organized as follows. Section 2 shortly analyzes the enabling technologies necessary to implement the chosen solution. Section 3 describes the relay selection mechanism based on MIH signaling. Then, Section 4 presents the simulation methodology used to assess the performance of the new algorithms. Results are presented in Section 5. Finally, conclusions of the study are commented in Section 6.

\section{Current technological scenario}

In order to use MIH signaling to manage the routing of packets dynamically it is necessary to define a set of technological tools that support the proposed solution. The following sections define the basis of these technologies.

\subsection{LTE-advanced}

$4 \mathrm{G}$ cellular systems are being designed to increase the coverage and user data rates. The spectrum for $4 \mathrm{G}$ systems can be distinguished into five bands: the $450 \mathrm{MHz}$ band, the digital dividend (DD) band around $700 \mathrm{MHz}$ (spectrum available after the switchover from analog to digital television), advanced wireless services (AWS) band between $1.7-2.1 \mathrm{GHz}$, the $2.5 \mathrm{GHz}$ and, finally, the $\mathrm{C}$ band around $3.5 \mathrm{GHz}$ [12]. As can be seen, most of these bands are above $2 \mathrm{GHz}$, where propagation loss exponent is higher and therefore, under these conditions, traditional cellular architectures require a higher density of BSs. Obviously, increasing the density of BSs is very costly for operators and other alternatives are preferred. One solution may be to increase the allocated bandwidth or spectral efficiency. Indeed, this article will show that mobile relaying implies a significant increase in spectral efficiency.

In a relay scenario there are three types of link: a BS to user link, a base station to relay link (also known as backhaul) and a relay to user link. It is worth noting that the relay node is wirelessly connected to the radio access network through the BS or donor cell. 3GPP distinguishes two types of architectures [13] focusing on fixed relays:

- Architecture A. This architecture is based on the termination at the relay of both U-Plane and CPlane protocols of the S1 interface. In this proposal, the relay can be seen as a BS for the user.

- Architecture B. In this case the donor BS terminates S1 connections towards Evolved Packet Core 
(EPC) and the relay node can be seen as a cell managed by the donor BS from the EPC and neighbor BSs point of view. In this architecture, some legacy MAC/RLC/PDCP protocols would need to be modified.

\subsection{IEEE $802.11 \mathrm{n}$}

One of the most pervasive wireless technologies for use in homes, offices, and other multiple scenarios is the IEEE 802.11 technology. 802.11n is the version that offers more peak data rate, reaching more than $100 \mathrm{Mbps}$. The used bandwidths are 20 or $40 \mathrm{MHz}$ in the bands of 2.4 or $5 \mathrm{GHz}$ [14]. The physical layer data rate can even exceed 300 Mbps provided $2 \times 2$ spatial multiplexing and $40 \mathrm{MHz}$ bandwidth. This high performance is achieved through a range of new technological solutions such as: Multiple Input Multiple Output (MIMO) schemes, Spatial Multiplexing (SM), spatial mapping (including beamforming), Space-Time Block Coding (STBC), and Low-Density Parity Check (LDPC) coding.

\subsection{Media independent handover}

The IEEE 802.21 MIH is specially designed to perform handovers between different IEEE 802 architectures (802.3, 802.11, 802.16) [8]. However, other 3GPP technologies such as LTE-A can be included in its operation. Next generation handsets are ideal candidates for installing and using the MIH protocol since they have multiple radio access interfaces (UMTS, LTE, WiFi, etc.). Moreover, MIH protocol can easily be included in the application layer.

Mainly, MIH is based on the exchange of messages reporting a subset of PHY/MAC layer events. The MIH functions are enabled by an entity called MIH Function (MIHF), which provides MIH Event Services (MIES), MIH Command Services (MICS), and MIH Information Services (MIIS). Figure 1 shows the main entities in the $\mathrm{MIH}$ protocol, as well as the events and commands generated by these entities. The MIES detects changes in the link layer and initiates events from both local and remote interfaces. The MICS offers the MIH-User control over the connection properties that are relevant to the handover. Finally, the MIIS provides information on different heterogeneous networks. There are specific events to notify link power going down, link disconnection, degradation of the channel, link handover is imminent, and so on.

The different events and commands are defined in the MIH standard. Depending on where the information is originated, the MIHF entity could receive or transmit reports on the configuration and condition of the radio access networks the $\mathrm{MN}$ is detecting. If the information is obtained remotely, the local MIHF entity receives information from the remote MIHF entity that is located in the network. However, when information is received from the lower layers of the protocol stack, this is obtained through service primitives that define the interface. For example, if the signal strength received by a remote entity falls below a threshold, then lower layers detect it and send a Link Going Down event to the MIHF entity. This entity communicates with the remote MIHF through Remote MIH Events. Once the event reaches the local MIHF entity, it is forwarded via a MIH event to the MIH-User. In the same way as with events, local MIHUser can send a command to make lower layers perform a specific operation. For example, thresholds can be adjusted, the active link quality can be measured in terms of Signal to Interference plus Noise Ratio (SINR), Bit Error Rate (BER), etc. Finally, to integrate MIH within 3GPP systems there is no need for new protocols to access MIHF services. These services can be mapped to those already existing in 3GPP [8].

\subsection{Localization}

User positioning can significantly reduce the complexity of the relay selection process. LTE-A specification considers MN localization through the LTE Positioning Protocol (LPP) and LPP Annex (LPPa) [15,16]. Several different positioning methods are mentioned in the standard, namely: Observed Time Difference of Arrival (OTDoA), Assisted-Global Navigation Satellite System (A-GNSS) and Enhanced-Cell ID (E-CID). Implementation details are omitted here but the interested reader can refer to the standard for further information. All of these positioning methods are based on measurements collected by the MN or the BS. The Mobility Management Entity (MME) is the entity that receives the request for the localization of a MN from another entity such as a MN, BS or other nodes. Then, the MME sends a location service request to the Enhanced Serving Mobile Location Centre (E-SMLC), which will execute the positioning procedure through LPP and LPPa protocols. The SLs interface defined between E-SMLC and MME serves as a tunnel for the E-SMLC to transparently carry LPP and LPPa protocols through the MME, in addition to transport the Location Services Application Protocol (LCS-AP) messages and parameters. The study of different positioning methods is outside the scope of this article. However, it is worth noting that in LTE-A advanced location methods are available and can be used by the relay selection mechanism proposed in this article.

Concerning the signaling overhead of user positioning, note that the periodicity of LPP methods must be higher than $0.5 \mathrm{~s}$, which is the typical period of measurement reports sent by Long Term Evolution (LTE) mobile nodes. Considering that the MIIS entity is independent for each BS, the data base burden is limited to hundreds of inserts per second, which is an order of magnitude 


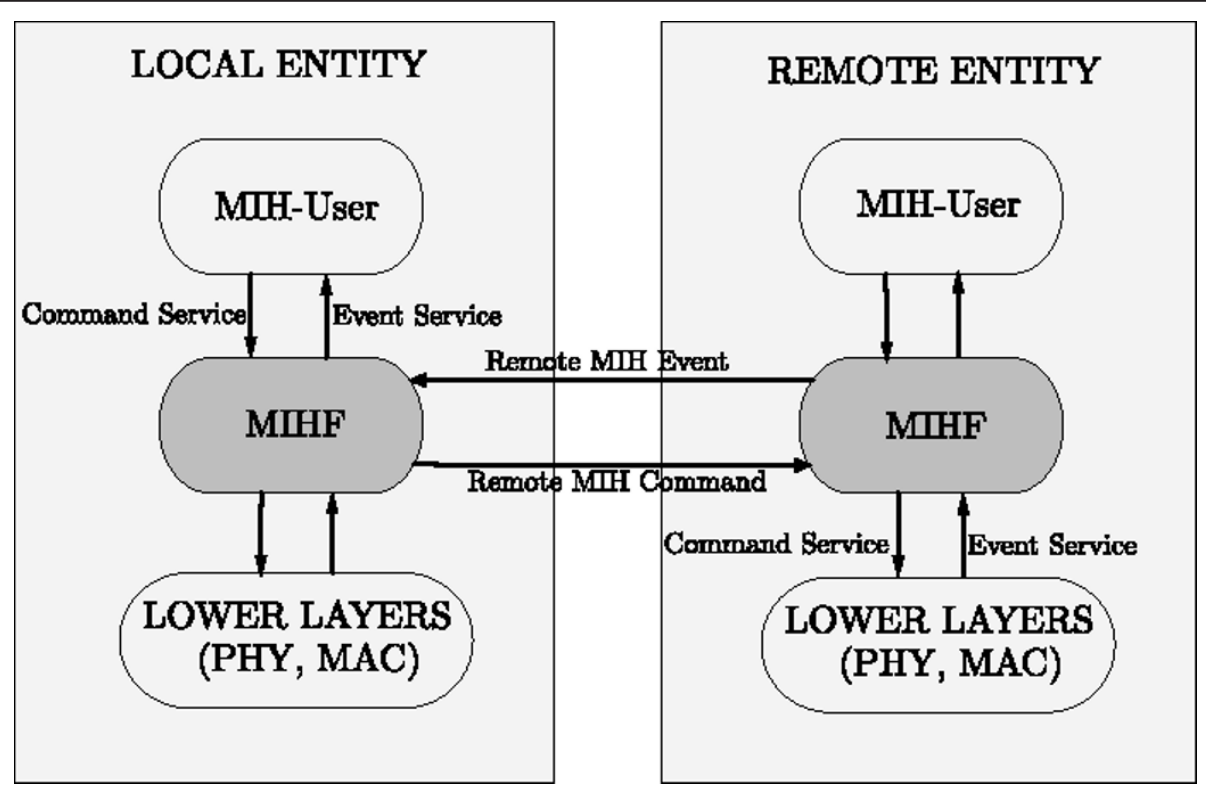

Figure 1 Media independent handover operation

lower than the current capacity of commercial data bases

\section{MIH-driven relay selection mechanism (MIDRES)}

Cell-edge users suffer a reduction in data rates as compared with users who are close to the BS because the SINR is usually lower. This causes some unfairness among nodes due to their location. Moreover, considering that the spectral efficiency requirements set by the International Telecommunication Union (ITU) for 4G technologies are very demanding [12], both IEEE and 3GPP have decided to use relays to increase the celledge user spectral efficiency. The use of relays decreases the transmission range, increasing the probability of receiving the data correctly, allowing higher data rates than those achieved without relays.

Figure 2 shows the scenario under study. Three types of nodes are distinguished: a BS, MNs, and MRs. The $\mathrm{MN}$ that is experiencing bad channel conditions is referred to as Solicitor Mobile Node (SMN). In this scenario there exist MNs receiving from the BS (active nodes) and MNs in the idle mode (passive nodes), which are the only ones that can relay signals from or to the SMN. Both MNs and MRs have two interfaces: an LTE-A interface to communicate directly with the BS and a WiFi interface for the MN to MR link. It is considered that the link between the MR and the MN is implemented with IEEE 802.11n standard [14]. At baseline, all MNs are connected with LTE-A unless the use of mobile relaying implies higher data rates.

The MIH-Driven Relay Selection Mechanism (MIDRES) proposed in this article uses MIH signaling to define a routing and handover mechanism for mobile relaying in Fourth Generation (4G) mobile systems. In this article, it is assumed that nodes can have both LTE$\mathrm{A}$ and Wireless Fidelity (WiFi) radio interfaces simultaneously active. When handover occurs, the packet route switches from one technology to another and the old technology transits to the idle state. As shown in Figure 2, if a MN connected to LTE-A experiences a SINR level below the quality threshold, $T h_{L T E}$, during Time to Trigger (TTT) seconds, then a Link Going Down (LGD) event is sent from lower layers to the MIH-User situated in upper layers. Similarly, a LGD event could be initiated and sent to the MIH-User in the MR. These events initiate the handover procedure.

It is assumed that all MNs and the BS are MIH-Users and implement MIHFs. In the BS the MIHF is also known as Point of Service (PoS). As shown in Figure 1, $\mathrm{MIH}$ communication among entities such as BSs or MNs is carried out through MIHFs. The destination of an event is established with a subscription mechanism that enables MIH-Users to subscribe to particular event types located either in the same equipment or in remote nodes. The main difference between the MIHFs of BSs and MNs is that MNs announce events from lower layers to the subscribed entities whereas the BS initiates and manages possible handovers among technologies.

In mobile relaying three different situations may occur:

1. Situation 1. The SMN is connected to LTE-A and the SINR level is lower than $T h_{\mathrm{LTE}}$. In this case, MIDRES initiates the handover procedure to select the most appropriate MR. 


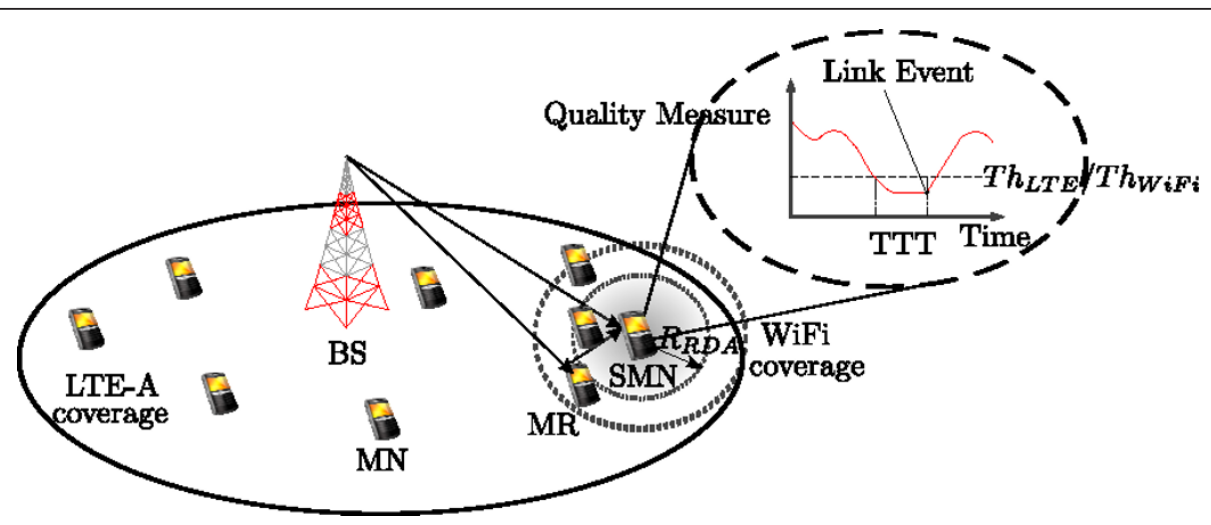

Figure 2 Simulation scenario under study.

2. Situation 2. The mobile relaying for a $M N$ is active and either the LTE-A or the WiFi interface goes below the quality threshold. Then, MIDRES initiates the procedure looking for a new available MR or making a handover back to LTE-A.

3. Situation 3. The mobile relaying for a $\mathrm{MN}$ is active and the LTE-A SINR level of the MN is higher than the quality threshold. It is considered in this article that this threshold is again $T h_{\mathrm{LTE}}$. In this case, MIDRES initiates the procedure searching for the best connection configuration.

\subsection{Situation 1}

Figure 3 shows the exchange of messages when mobile relaying provides better throughput to the SMN than the direct LTE-A link. The figure also shows the connection state for LTE-A and WiFi. In this situation, MIDRES procedure includes the following phases:

1. Monitoring and notification. When the SMN detects that the SINR level is below $T h_{\mathrm{LTE}}$ during TTT seconds, the lower layers send a LGD event to the MIH-User that forwards this event to the BS with the Link_Parameters_Report.indication message.

2. Information query. The BS MIH-User sends a Get_Information.request message towards the Information Server (IS) asking which relay nodes are candidates. The BS could attach a radius called $R_{\mathrm{RDA}}$, centered at the indicated location. Otherwise, the IS would decide upon this radius. At this point, the IS can run the proposed Relay Discrimination Algorithm (RDA) to reduce MIH signaling. Basically, the RDA consists in selecting those cooperative MNs that are at a distance less than $R_{\mathrm{RDA}}$ from the SMN. Note that the IS can periodically estimate the location of all MNs using E-SMLC described in Section 2.4. Finally, the IS entity responds with a Get_Information.response message that includes the list of candidate MRs.
3. Resource availability check. After the confirmation of available relays, the BS sends a $\mathrm{MIH}_{-}-$ Net_HO_Candidate_Query.request to the $\mathrm{MN}$ providing the list of candidate MRs. The MN indicates the required resources in the $\mathrm{MIH} \_\mathrm{Net}_{-} \mathrm{HO}_{-}-$ Candidate_Query.response message. At this stage the SMN activates its WiFi interface and sends periodic HELLO messages so that the candidate MRs can detect its presence. Next, the BS polls all MR candidates on the feasibility of the connection by sending a MIH_N2N_HO_Query_Resources.response. Once the candidates receive this message, they activate their WiFi interface and measure the link quality with the SMN, analyzing the received HELLO messages power and therefore the link throughput. After this measure, each relay node sends the result to the BS in a MIH_N2N_HO_Query Resources.response message through the LTE-A interface. Note that, in addition to this information, the BS can estimate the mean throughput of its link with the MR since the LTE-A connection is active at this stage. Using these data, the BS decides on the best connectivity option, that is, remain connected to LTE-A or use a MR.

4. Resource Preparation. If the best option is to use a relay then the process continues. The BS sends a MIH_N2N_HO_Commit.request message to the selected MR marking the beginning of the routing process. The relays answers with a MIH_N2N_HO_Commit.response. From this point on, the relay will route all packets from the BS to the SMN. For all purposes, the relay will be connected with the BS as another user. To distinguish the final destination (it may be that the relay receives a packet for itself), the relay will only have to inspect the IP packet's destination address. Of course, for this to be viable, it is necessary that the relay possesses routing capabilities. In the same way, the BS informs the SMN about the decision with a MIH_Net_HO_Commit.request message to 


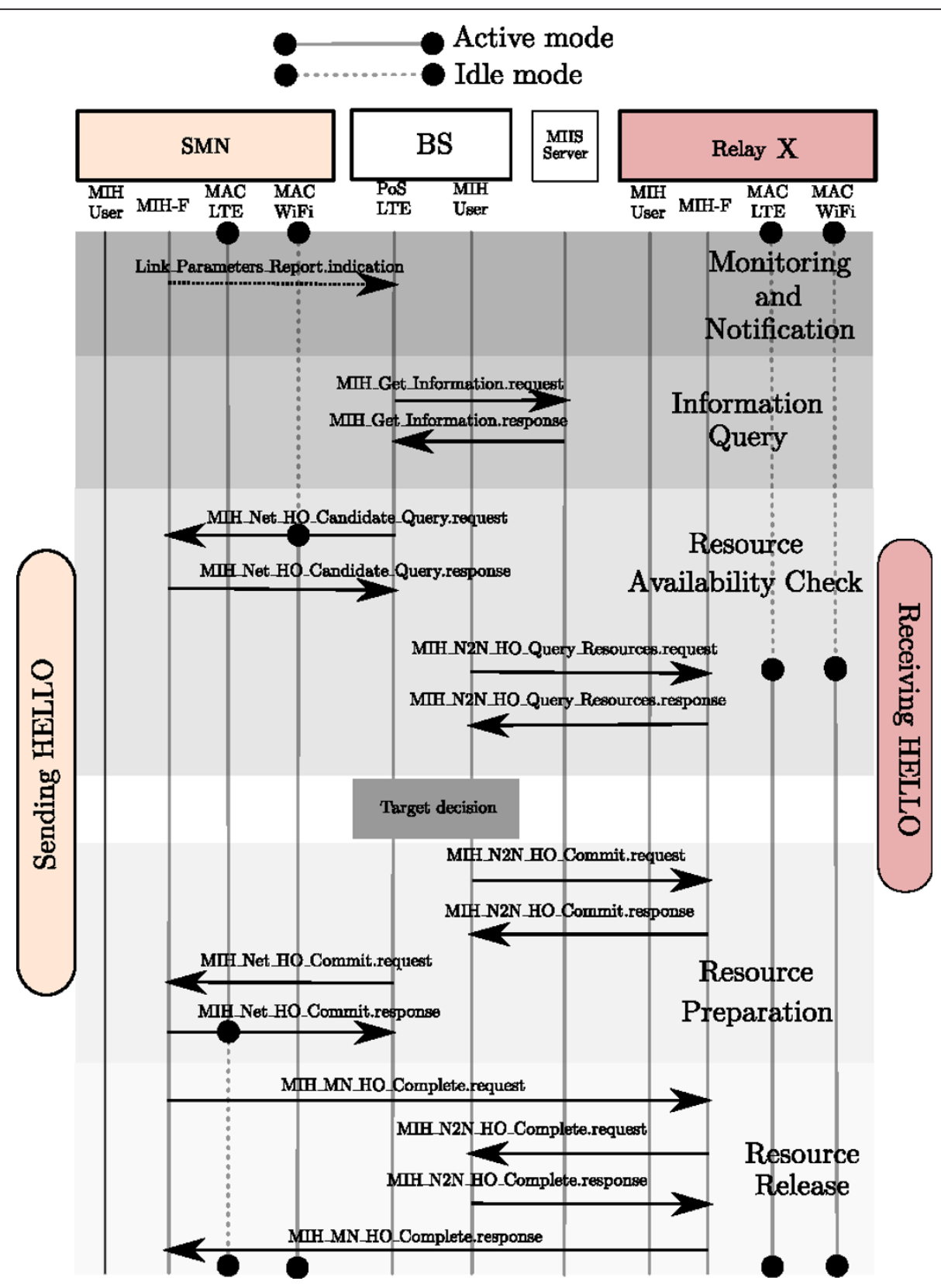

Figure 3 MIDRES procedure to configure a MR.

commit handover towards the specified relay. The new radio interface connection is established and the $\mathrm{MN}$ sends a MIH_Net_HO_Commit.response to the BS.

5. Resource Release. In the last stage mobile relaying must be confirmed. The SMN sends a MIH_MN_HO_Complete.request to the selected relay that answers with a MIH_MN_HO_Complete.response. The completion is also reported to the BS with a MIH_N2N_HO_Complete.request and its corresponding response message.
At this point it is worth discussing about another alternative of MIDRES in which the SMN measures the quality of the candidate MRs. Note that the decision of making the MRs measure the channel is aimed at reducing the handover delay. With the current proposal of MIDRES, the SMN is the only one sending HELLO messages and this reduces the contention problems. With the other alternative, all relay nodes should content to seize the channel and therefore there would be potential collisions and hidden node problems. As a 
consequence the time required for the handover will be much higher.

\subsection{Situation 2}

Figure 4 shows the exchange of messages when, in an active mobile relaying, the power level of the WiFi radio interface falls below the threshold, $T h_{\mathrm{W}_{\mathrm{iFi}}}$. Note that the procedure would be the same in case of degradation of the LTE-A link. The MR sends to the BS the corresponding Link_Parameters_Report.indication message. From this moment all phases of the MIDRES procedure are identical to the Situation 1, except the resource release phase. Now the BS sends a MIH_N2N_HO_Complete. request to the old relay to release the assigned resources. The old relay answers with a MIH_N2N_HO_Complete. response. Note that the procedure also contemplates the possibility of the direct LTE-A link being the most convenient one. In this case, the $\mathrm{MN}$ sends the MIH_MN_HO_Complete.request message directly to the BS.

\subsection{Situation 3}

When the LTE-A SINR level at the SMN is higher than the quality threshold, an event is sent from its lower layers to the MIH-User. This Link-Up event is notified by the SMN to the BS through a Link_Parameters Report.indication message. The signaling procedure is the same as in situation 2 with the possibility of not selecting any new MR.

\section{Assessment methodology and system modeling}

Assessment methodology in this study was based on system level simulations. The baseline for the simulation methodology and system modeling are the guidelines

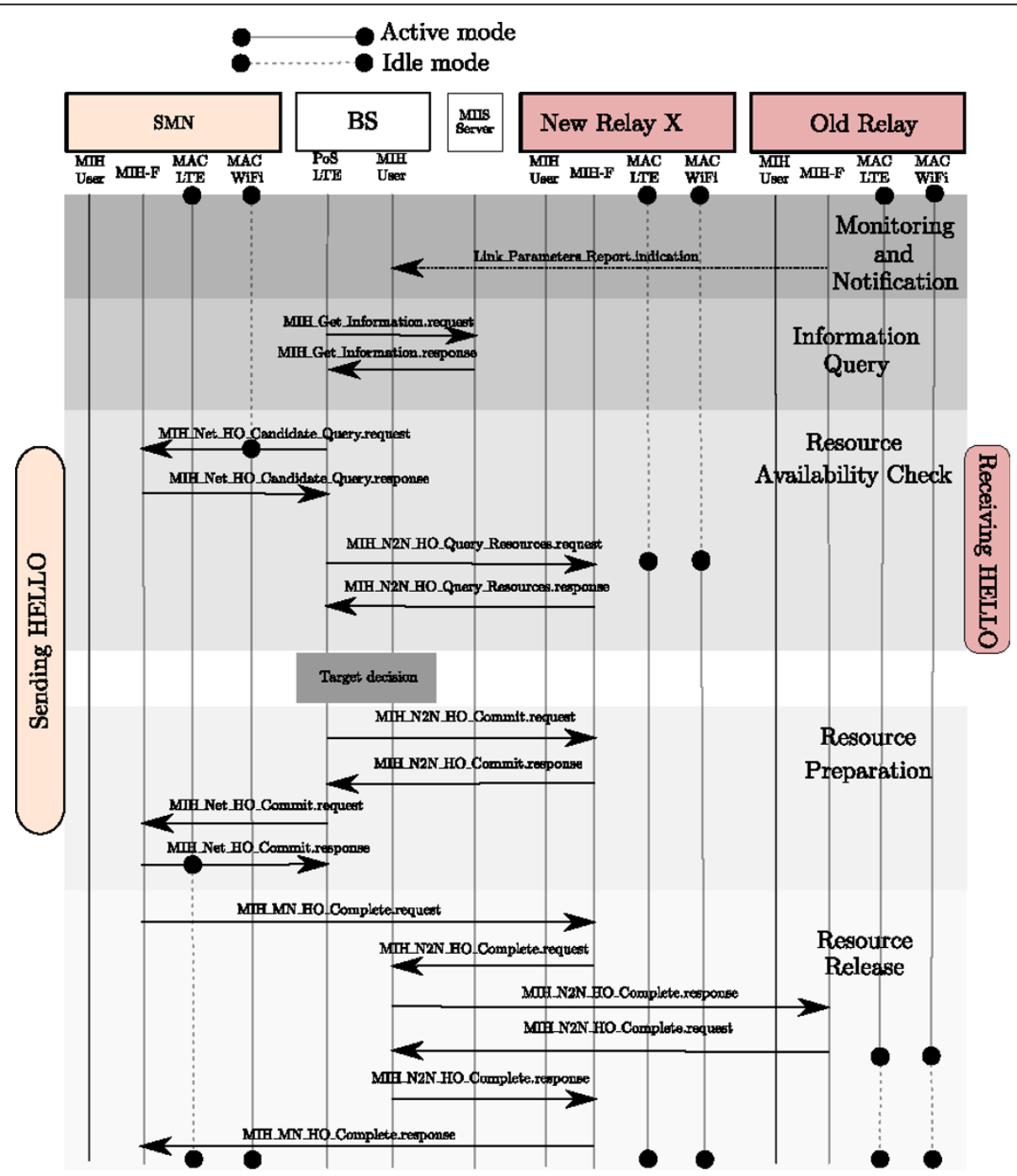

Figure 4 MIDRES procedure to change the MR. 
provided by [17] for the evaluation of International Mobile Telecommunications Advanced (IMT-Advanced) technologies. Nevertheless, some simplifications have been considered in order to reduce the complexity of the methodology proposed in [17].

A simple network layout was assumed with an isolated cell with a unique BS in its center that serves a circular area with radius $R$. A population of MNs is spatially uniformly distributed within the cell area. The initial position of a user $u$ is randomly taken in polar coordinates from two uniform distributions, being the radius, $r_{u}=\sqrt{U\left[0, R^{2}\right]}$ and the angle $\alpha_{u}=U[0,2 \cdot \pi]$.

Although in this model the cell layout deviates from the original one specified in [17], the same SINR Cumulative Density Function (CDF) as in the original layout is found in the cell of the new layout. This is achieved thanks to a position-SINR relation model specifically designed for this purpose. Using a complete simulator, with the original layout, the received SINR CDFs after antenna receiver were obtained for the different evaluation scenarios: Indoor hotspot ( $\mathrm{InH})$, Urban microcell (UMi), Urban macrocell (UMa), and Rural Macrocell (RMa). Then for each user, position has a one-to-one correspondence with the SINR value according to the following formula:

$$
\operatorname{SINR}_{u}=\mathrm{CDF}^{-1}\left(1-\left(\frac{r_{u}}{R}\right)^{2}\right),
$$

where $C D F$ is the SINR CDF for any particular scenario, and $C D F^{-1}$ is its inverse. It is worth noting that in this model the higher SINR values are obtained for the lower distances to the BS.

Multipath fading is not emulated in this assessment. Then, SINR values represent wideband measures. In LTE-A, channel capacity was obtained for each user translating the wideband SINR value to a throughput value through Look-Up Table (LUT) calculated in link level simulations. Different LUT were obtained for each evaluation scenario and MN multi-antenna configuration. Similar throughput vs wideband SINR curves are found in [2]. Both, the complete system level simulator and the link level simulator were validated in the framework of the Wireless World Initiative New Radio + (WINNER+) project [18] being used in the IMTAdvanced candidate evaluation carried out within ITUR. Concerning WiFi, channel capacity is a function of distance between transmitter and receiver as described in [19].

MNs are in constant motion throughout the simulations with a fixed speed and follow a model similar to the Random Waypoint Model [20]. The MN path comprises a sequence of movement intervals in which direction is fixed. In each movement interval the starting point is the ending point of the previous interval, while the ending point is drawn from a spatially uniform distribution over the cell area.

MNs can be in two states and do not change this state during the simulation. MNs in idle state can relay signals while MNs in active state are receiving data and cannot relay signals. Concerning the traffic model, for the sake of simplicity a Constant Bit Rate (CBR) traffic source has been used. With respect to scheduling, LTEA performs a proportional fair allocation of resources.

Finally, concerning MIH signaling, it is worth noting that the simulation tool models the MIH messages and accounts for the overhead that this additional signaling entails. Only the MIH subscription messages that can be originated both locally and remotely have not been considered in the signaling burden. Concerning handover delay, the simulation takes into account the idle to connected mode latency in LTE-A and all the time required in the discovery and connection phase of the WiFi interface.

Additional assumptions of the simulation methodology are indicated in Table 1.

\section{Results and discussion}

MIDRES was designed to make MIH protocol compatible with LTE-A system. With this aim, it is necessary to perform an optimal threshold setting to find a trade off between channel quality improvement and signaling overhead. Hence the importance of Section 5.1. On the other hand, MIH signaling can cause a performance hit as the number of candidate relay nodes increases since signaling overhead increases too. Section 5.2 presents some results concerning the RDA algorithm that reduces the amount of candidate relays and, consequently, the signaling overhead. Next, the effect of the MN speed on the performance of mobile relaying is

\begin{tabular}{|c|c|}
\hline Scenarios/speed $(\mathrm{km} / \mathrm{h}) /$ radius (m) & $\begin{array}{c}\operatorname{lnH} / 3 / 20 \text { UMi/3/67 } \\
\text { UMa/30/167 RMA/120/577 }\end{array}$ \\
\hline Number of active nodes & 10 \\
\hline Number of idle nodes & 50 \\
\hline Packet size (bytes) & 128 \\
\hline Application rate (Mbps) & $4(\mathrm{InH}) / 2(\mathrm{UMi}, \mathrm{UMa})$ \\
\hline TाT (ms) & 100 \\
\hline WiFi carrier frequency (GHz) & 2.4 \\
\hline WiFi bandwidth $(\mathrm{MHz})$ & 20 \\
\hline WiFi transmission power (dBm) & 15 \\
\hline WiFi antenna configuration & $1 \times 1$ \\
\hline LTE-A antenna configuration & $1 \times 2$ \\
\hline LTE-A bandwidth (MHz) & 10 \\
\hline
\end{tabular}


evaluated in Section 5.3. Finally, Section 5.4 is devoted to the study of cost savings that result from using mobile relaying in LTE-A.

\subsection{Threshold evaluation}

The study of the quality threshold set by the MIH-User is of paramount importance to reach an equilibrium between signaling load and system performance. There exist three different thresholds:

1. WiFi threshold $\left(T h_{\mathrm{W}_{\mathrm{iFi}}}\right)$ : If the WiFi link falls below this threshold the MR replacement process starts.

2. LTE-A threshold $\left(T h_{\mathrm{LTE}}\right)$ : This threshold activates the search of a MR to support the connectivity.

3. LTE-A reactivation threshold: If the LTE-A signal surpasses this threshold then the SMN could be back to the single-hop LTE-A communication.

For the sake of simplicity, in this article $T h_{\mathrm{WiF}_{\mathrm{iF}}}$ is set to $-82 \mathrm{dBm}$ and the reactivation threshold equals $T h_{\mathrm{LTE}}$. Therefore, this section only focused on the optimization of the LTE-A threshold based on, firstly, the minimization of the number of required relays and, secondly, the maximization of the Cell-edge User Throuhput (CEUT).

Figure 5 evaluates the Mean User Throughput (MUT) with an increasing number of MR available in the scenario. Note that 0 MR represents the scenario without mobile relaying. Two scenarios (InH and UMi) and four thresholds are depicted. The number of active nodes in the cell is 10 following the guidelines provided by [17].

It can be observed that mobile relaying improves remarkably system performance. In the InH scenario, the higher the threshold is the better the performance. It is always beneficial to use a MR since, in such a small scenario, the WiFi interface exhibits higher data rates and reducing the hop length in LTE-A also improves performance. However, there is an optimum in the number of MRs, which in the InH case is around 10. With more than 10 MRs the additional diversity in the selection of the best MR does not compensate the increase in signaling. To sum up, for small scenarios the LTE-A threshold must be set as high as possible but the candidate set must be restricted to 10 .

However, for wider scenarios the selection is more challenging. As an example, this article analyzes the UMi case. If $T h_{L T E}$ is too small, the system does not benefit from the availability of relays. In fact, with a small number of available relays it is better to choose a lower threshold since with higher probability the relay will not be beneficial for the link. On the contrary, if $T h_{\mathrm{LTE}}$ is too high, with higher probability the WiFi link would be worse than the LTE-A link and the system would waste resources on useless signaling. Therefore, an in-depth analysis is needed.

Figure 5 shows that the minimum number of relays to get good diversity is around 20. Note that this number is higher than in the InH case, since more relays are required to have the same diversity in the selection of the best MR. On the other hand, Figure 6 shows the MUT CDF for this required minimum number of MRs. In the UMi scenario the optimum threshold changes depending on the objective. In order to maximize the CEUT (5th percentile of the $\mathrm{CDF}$ ), the best threshold is between 10 and $15 \mathrm{~dB}$. In fact, the optimum is $11 \mathrm{~dB}$ as shown in Table 2 .

As a guideline, for the UMa scenario the required minimum number of MRs is around 25 and the optimum threshold is $12 \mathrm{~dB}$. Concerning the RMa scenario, the number of relays is around 35 and the optimum threshold is again $12 \mathrm{~dB}$.

\subsection{Performance evaluation of the relay discrimination algorithm (RDA)}

The Relay Discrimination Algorithm (RDA) is useful for reducing the MIH signaling. Without the use of this

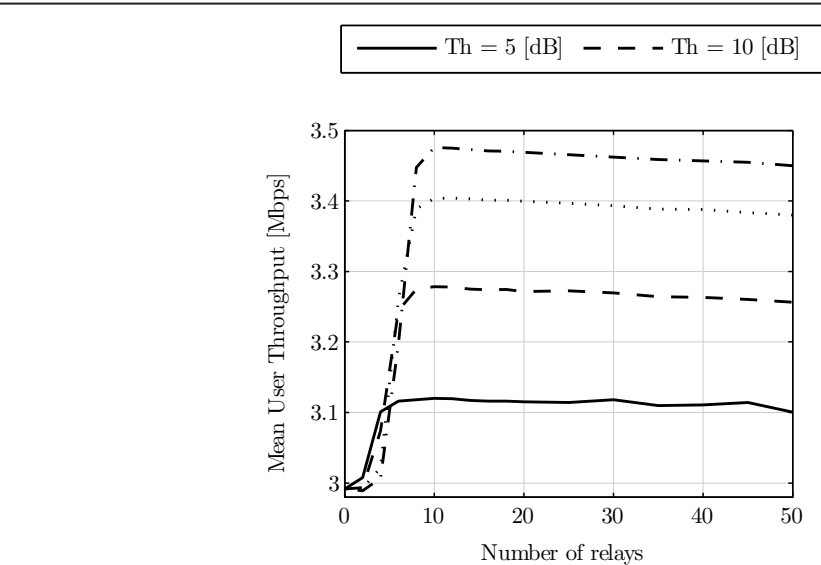

$\ldots \ldots \mathrm{Th}=15[\mathrm{~dB}] \quad \cdot \boldsymbol{-} \cdot \mathrm{Th}=20[\mathrm{~dB}]$

Figure 5 Threshold analysis for InH (left) and UMi (right) scenarios.

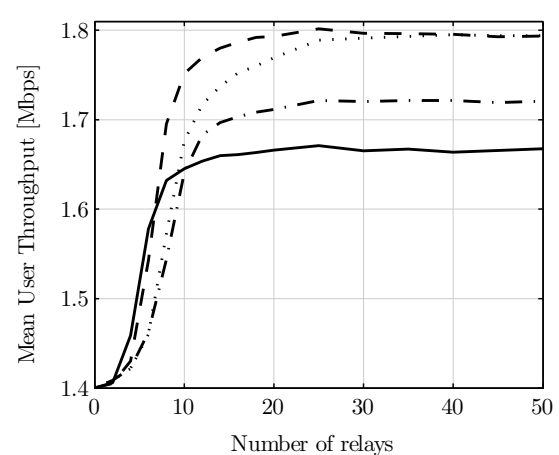



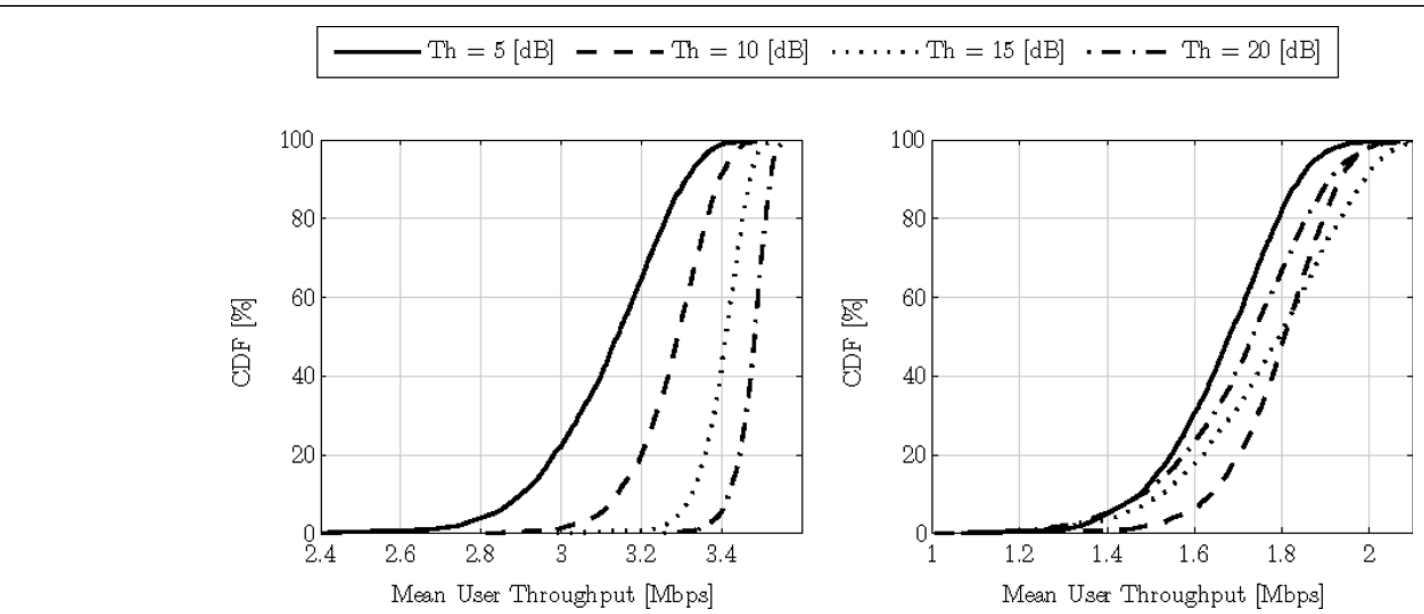

Figure 6 CDF of the mean user throughput for InH (left) and UMi (right) scenarios.

algorithm by the MIIS server, the BS would send a MIH_N2N_HO_Query_Resources.request to all idle nodes. As the number of cooperative nodes increases the MIH protocol could be unfeasible. Thus, RDA algorithm only requires that the MIIS Server knows the MNs location. As discussed in Section 2.4, LTE-A standard encompasses several positioning methods that can be used for this purpose.

In this section, a UMa scenario is assumed with $T h_{\mathrm{LTE}}=$ $12 \mathrm{~dB}$. Figure 7 shows the MUT varying the parameter $R_{\mathrm{RDA}}$ for an increasing number of MRs. It can be seen that, when restricting the search area, a larger number of cooperative relay nodes is required to get the same MUT since the probability of a relay being in the search area is smaller. However, when the number of available relays increases, there is an increment in the signaling load that can be reduced with the RDA. In fact, the optimum value of $R_{\mathrm{RDA}}$ depends on the number of available MRs as shown in Figure 7. For a higher number of cooperative nodes a smaller search radius must be used, since this reduces the signaling overhead.

\subsection{Velocity impact evaluation in performance}

This section evaluates the effect of the MN speed on the mobile relaying performance. A UMa scenario was simulated in which user speed augmented from 40 to $90 \mathrm{~km} / \mathrm{h}$. Figure 8 shows that the performance of mobile relaying deteriorates as the user speed increases. This is due to the fact that the mobile relaying links are less stable and signaling and handover delay degrade the MUT. However, even with $90 \mathrm{~km} / \mathrm{h}$ mobile relaying is preferred as

Table 2 CEUT for different $T h_{\mathrm{LTE}}$

\begin{tabular}{lcccccc}
\hline$T h_{\text {LTE }}[\mathrm{dB}]$ & $\mathbf{1 0}$ & $\mathbf{1 1}$ & $\mathbf{1 2}$ & $\mathbf{1 3}$ & $\mathbf{1 4}$ & $\mathbf{1 5}$ \\
\hline CEUT [Mbps] & 1.5797 & 1.5916 & 1.5630 & 1.5524 & 1.5098 & 1.4377 \\
\hline
\end{tabular}

compared with the conventional single-hop scenario. This is due to the fast-switching capacity of MIDRES and the good coverage of WiFi in outdoor.

\subsection{Deployment cost in an LTE-A system}

LTE-A systems are designed to increase end user data rates as compared with Third Generation (3G) systems. These high data rates may only be offered to users near the BS, creating a situation of unfairness between MNs. However, operators aim to offer higher rates to as many users as possible with the least possible cost. The use of MRs is considered as a key factor to increase the coverage thus deploying a lightweight wireless system.

A simplified high level financial analysis of the LTE-A deployment was performed to assess the reduction of costs that can be achieved through the use of MRs. This study was carried out for a fictive region of an area of 200 square kilometers similar to that described in [21], which represents a typical European city scenario. Although the

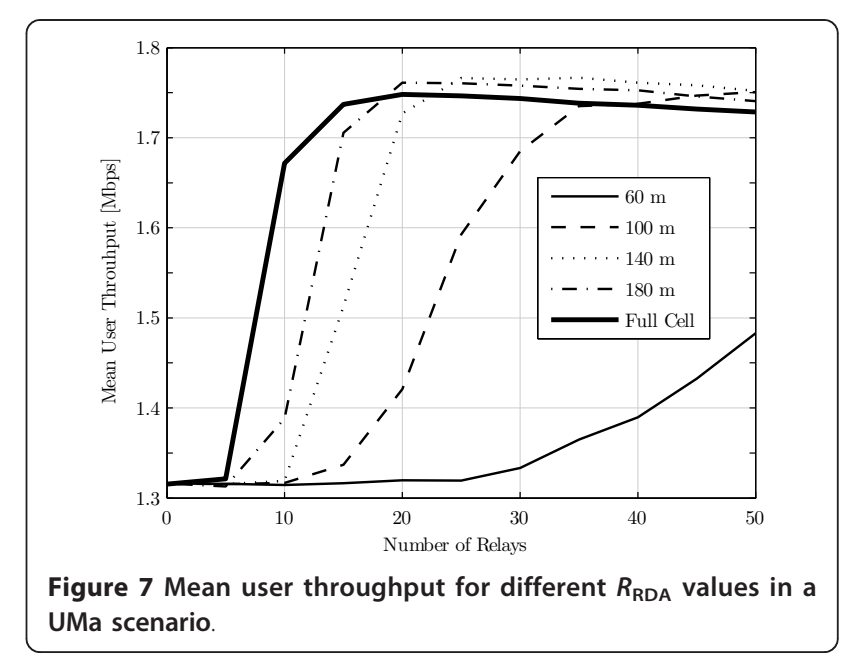




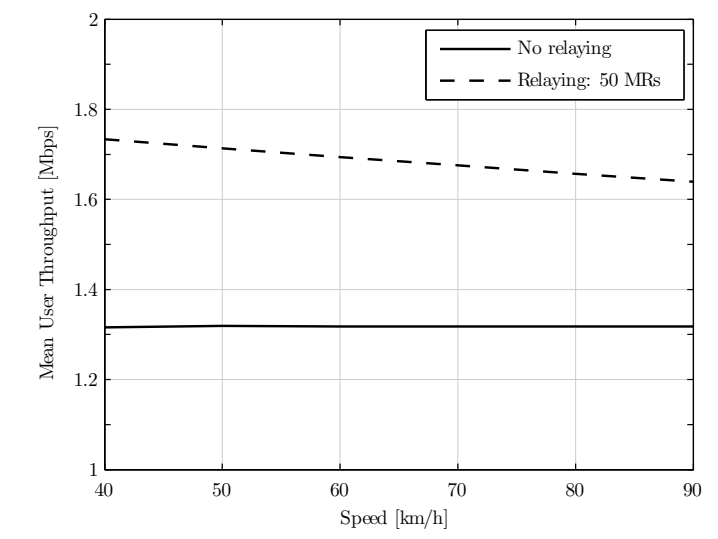

Figure 8 Mean user throughput for different values of MN speed in a UMa scenario.

total population was assumed to be 500,000 people, the LTE-A network was expected to cover only $70 \%$ of the population. In particular, according to [21], the number of LTE-A subscribers served by one operator in this fictitious scenario was predicted to be, at most, 55,000 by the third year. That is to say, a population density of 275 people per $\mathrm{km}^{2}$ is considered.

In order to study the real economical impact of mobile relaying, a quality criterion for Cell-Edge User Spectral Efficiency (CEUSE) of $0.12 \mathrm{~b} / \mathrm{s} / \mathrm{Hz}$ was fixed. Multiple simulations were performed to assess the number of base stations required to achieved such quality criterion in a scenario with the above-mentioned population density for different cell radius configurations with and without relaying. A UMa scenario was considered. For each cell radius configuration, the proper number of total users was calculated according to the fixed population density. The number of active MNs in each cell was $1 / 3$ of this total number, being the rest of users in idle state. The number of possible MRs in each cell is a percentage of the number of idle users. The considered percentages range from $0 \%$ (network without relaying) to the $100 \%$ in steps of $20 \%$. In all simulations $T h_{\mathrm{LTE}}=12 \mathrm{~dB}$.

As shown in Figure 9, a network without relaying reaches the quality criterion with a cell radius of $192.35 \mathrm{~m}$, whereas with a percentage of $80 \%$ among idle nodes, coverage increases by $7.46 \mathrm{~m}$, which represents an increase of $7.91 \%$. With $100 \%$ of relay nodes coverage increases $19.35 \mathrm{~m}$, that is, a $21.16 \%$. Note that without using relaying and until a percentage of $60 \%$ of idle nodes, the CEUSE is almost equal for different cell radius.

For the financial analysis, Capital Expenditure (CAPEX) and Operational Expenditure (OPEX) per year were calculated. In the CAPEX analysis it was assumed that the mobile operator deploys LTE-A using the available infrastructure. Therefore, civil work expenditures were not taken into account, neither the cost of acquiring spectrum

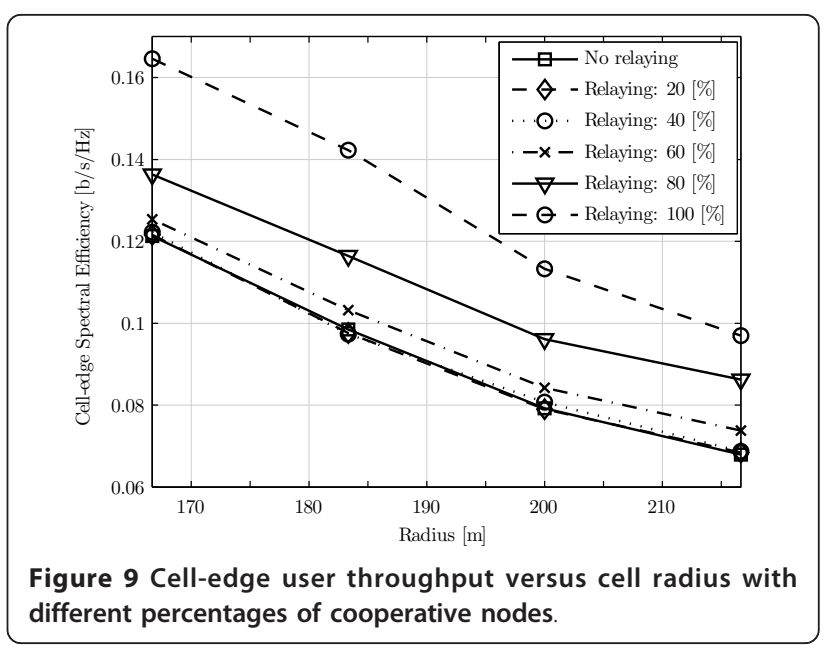

licenses, since operators may use frequency bands of current technologies to minimize the cost of LTE-A. Finally, CAPEX encompasses LTE-A equipment cost $(50 \mathrm{k} €$ per site [22]), labor-related deployment cost (10k€ per site) and upgrading the existing backhaul ( $2 \mathrm{k} €$ per site). The number of sites (composed of three cells in this study) multiplied by the investment cost per site yields the overall operator network investments. By combining the coverage area and the BS range, previously calculated, it is easy to obtain how many BSs are required.

On the other hand, OPEX accounts for site rental (10 k€ per site per year [23]), data backhaul costs ( $5 \mathrm{k} €$ per site [23]), maintenance ( $3 \mathrm{k} €$ per site [23]), marketing cost (1.85 $€$ per person [22]), administrative costs (addition of $10 \%$ of other running costs [22]) and the subsidy paid by the operator to reduce the price of new mobile terminals, thus facilitating the migration of subscribers $(160 €$ per subscriber [22]). Similarly, OPEX was calculated multiplying the number of sites by the running cost per site plus terminal subsidies, considering that the migration of subscribers to the new network occurs in progressive stages during these three years. Note that only one terminal replacement per user was supposed in this study. Moreover, OPEX is increased with the annuity payment of a loan for CAPEX requirements over this period at an interest rate of $4 \%$.

Table 3 shows the resulting deployment costs. For instance, according to the simulations, CAPEX varies from around $43 \mathrm{M} €$ with a basic LTE system to around $36 \mathrm{M} €$ when mobile relaying is added. This represents a $17.47 \%$ savings.

\section{Conclusions}

LTE has been designed as a future technology to cope with upcoming user requirements. This article has proposed a new relay selection mechanism based on the use of MIH signaling. This allows including mobile 
Table 3 Cost analysis of the LTE-A deployment

\begin{tabular}{lll}
\hline Scenario & CAPEX [M€] & OPEX [M€] \\
\hline LTE & 43.09 & 33.23 \\
LTE-A (80\%) & 39.93 & 31.07 \\
LTE-A (100\%) & 35.57 & 28.11 \\
\hline
\end{tabular}

relaying as an additional technique in an LTE-A system. The implementation of mobile relaying in LTE-A certainly enhances the overall system performance. In addition, the proposed relay selection procedure is a lowcost solution because no modifications of the LTE-A system architecture are required for its implementation.

However, the MIH-Driven Relay Selection Mechanism must be set up carefully and several aspects have been discussed throughout the article. First, SINR threshold that triggers the selection of relay must coincide with the point in which the cellular system is unable to cover user quality of service. Moreover, the selection of the relay entails additional signaling that must be reduced to a minimum to make the most of mobile relaying. A simple technique has been shown in this article to highlight the importance of this signaling overhead.

Finally, this article has shown the relevance of mobile relaying to reduce deployment costs. In an typical European city scenario, the reduction of CAPEX and OPEX is about $17.47 \%$ and $15.39 \%$, respectively.

\begin{abstract}
Abbreviations
3G: Third Generation; 3GPP: Third Generation Partnership Project; 4G: Fourth Generation; BS: Base Station; CBR: Constant Bit Rate; CDF: Cumulative Density Function; CEUSE: Cell-Edge User Spectral Efficiency; CEUT: Cell-edge User Throuhput; EPC: Evolved Packet Core; IEEE: Institute of Electrical and Electronic Engineers; IMT-Advanced: International Mobile Telecommunications Advanced InH: Indoor hotspot; IS: Information Server; ITU: International

Telecommunication Union; LGD: Link Going Down; LTE: Long Term Evolution; LTE-A: Long Term Evolution Advanced; LUT: Look-Up Table; MIDRES: MIHDriven Relay Selection Mechanism; MIH: Media Independent Handover; MIHF: MIH Function; MN: Mobile Node; MME: Mobility Management Entity; MR: Mobile Relay; MUT: Mean User Throughput; PoS: Point of Service; RMa: Rural Macrocell; SINR: Signal to Interference plus Noise Ratio; SMN: Solicitor Mobile Node; TT: Time to Trigger; UMa: Urban macrocell; UMi: Urban microcell; WiFi: Wireless Fidelity; WINNER+: Wireless World Initiative New Radio +; WiMAX: Worldwide Interoperability for Microwave Access.
\end{abstract}

\section{Acknowledgements}

This study was supported by the Spanish Ministry of Science under the project TEC2011-27723-C02-02.

The work of David Martín-Sacristán was supported by a FPU grant of the Spanish Ministry of Education.

\section{Competing interests}

The authors declare that they have no competing interests.

Received: 30 September 2011 Accepted: 26 March 2012

Published: 26 March 2012

\section{References}

1. ITU World Telecommunication/ICT Indicators database, Global ICT developments, 2000-2010. ITU Statistics (2010), http://www.itu.int/ict/ statistics. Accessed 20 March 2012
2. D Martín-Sacristán, JF Monserrat, J Cabrejas, D Calabuig, S Garrigas, N Cardona, On the way towards fourth-generation mobile: 3GPP LTE and LTEAdvanced. EURASIP J Wirel Commun Netw. 2009, 10 (2009)

3. 3GPP TR 36.913 V10.0.0, Requirements for further advancements for Evolved Universal Terrestrial Radio Access (E-UTRA) (LTE-Advanced) (Release 10). Technical Specification Group Radio Access Network (2011)

4. IEEE 802.16j, IEEE Standard for Local and metropolitan area networks Part 16: Air Interface for Broadband Wireless Access Systems. Amendment 1: Multi-hop Relay Specification (2009)

5. L Xiao, TE Fuja, DJ Costello, Mobile relaying: coverage extension and throughput enhancement. IEEE Trans Commun. 58 (2010)

6. K Vanganuru, M Puzio, G Sternberg, K Shah, S Kaur, Uplink system capacity of a cellular network with cooperative mobile relay, in Proceedings of the Wireless Telecommunications Symposium, 1-7 (2011)

7. $\mathrm{Z} \mathrm{Li}, \mathrm{H}$ Shen, Game theoretic analysis of cooperation incentive strategies in mobile ad-hoc networks. IEEE Trans Mobile Comput (2011). doi: 10.1109/ TMC.2011.151

8. IEEE 802.21, Draft standard for local and metropolitan area networks: media independent handover services. IEEE P802.21, D9.0 (2008)

9. SJ Bae, MY Chung, J So, Handover triggering mechanism based on IEEE 802.21 in heterogeneous networks with LTE and WLAN, in Proceedings of the International Conference on Information Networking (ICOIN), 399-403 (2011)

10. J Seol, J Chung, IEEE 802.21 MIH based Handover for Next Generation Mobile Communication Systems, in Proceedings of the 4th International Conference on Innovations in Information Technology (IIT '07), 431-435 (2007)

11. D Bültmann, J Luo, E Schulz, Media independent handover enabled advanced mobility management and its functional mapping, in Proceedings of the International Conference on Communications, Circuits and Systems (ICCCAS 2008), 134-138 (2008)

12. ITU-R M.2133, Requirements, evaluation criteria and submission templates for the development of IMT-Advanced. REPORT ITU-R (2008)

13. 3GPP TR 36.806 V9.0.0, Relay architectures for E-UTRA (LTE-Advanced) (Release 9). Technical Specification Group Radio Access Network (2010)

14. IEEE 802.11n, Part 11: Wireless LAN Medium Access Control (MAC) and Physical Layer (PHY) Specifications. Amendment 5: Enhancements for Higher Throughput (2009)

15. 3GPP TS 36.355, LTE Positioning Protocol (LPP). Technical Specification Group Radio Access Network (2011)

16. 3GPP TS 36.455, LTE Positioning Protocol A (LPPa). Technical Specification Group Radio Access Network (2011)

17. ITU-R M.2135, Guidelines for evaluation of radio interface technologies for IMT-Advanced. REPORT ITU-R (2009)

18. WINNER+ Evaluation Group, http://projects.celtic-initiative.org/winner +MINNER+\%20Evaluation\%20Group.html. Accessed 20 March 2012

19. DB Green, AS Obaidat, An accurate line of sight propagation performance model for ad-hoc 802.11 wireless LAN (WLAN) devices, in Proceedings of the IEEE International Conference on Communications (ICC 2002). 5, 3424-3428 (2002)

20. DB Johnson, DA Maltz, in Mobile Computing, Dynamic source routing in ad hoc wireless networks, Chap. 5, ed. T. Imielinski, H Korth. (Kluwer Academic Publishers, The Netherlands, 1996), pp. 153-181

21. WiMAX Forum, A Comparative Analysis of Spectrum Alternatives for WiMAX Networks with Deployment Scenarios Based on the U.S. $700 \mathrm{MHz}$ Band. Wimax Forum Website (2008)

22. S Forge, C Blackman, E Bohlin, The demand for future mobile communications markets and services in Europe. IPTS Technical Report Prepared for the European Commission-Joint Research Centre (2005)

23. K Johansson, A Furuskar, P Karlsson, J Zander, Relation between base station characteristics and cost structure in cellular systems. IEEE PIMRC2004 (2004)

doi:10.1186/1687-1499-2012-117

Cite this article as: Cabrejas et al: Application of MIH for the lightweight deployment of LTE-advanced systems through mobile relaying. EURASIP Journal on Wireless Communications and Networking 2012 2012:117. 